\title{
Drying of mangoes applying pulsed UV light as pretreatment
}

\section{Braga, T.R. ${ }^{\text {a }}$; Silva, E.O. ${ }^{\text {; }}$ Rodrigues, S. ${ }^{\text {; }}$ Fernandes, F.A.N. ${ }^{\text {a }}$}

${ }^{a}$ Universidade Federal do Ceara, Departamento de Engenharia Química, Fortaleza - CE, Brazil

${ }^{\mathrm{b}}$ Embrapa Agroindústria Tropical, Fortaleza - CE, Brazil

${ }^{\text {c }}$ Universidade Federal do Ceara, Departamento de Engenharia de Alimentos, Fortaleza - CE, Brazil

*E-mail of the corresponding author: fabiano@ufc.br

\begin{abstract}
High intensity pulsed UV Light is a non-thermal treatment used in sanitization of fruits and vegetables. In this work, we have applied high intensity pulsed $U V$ light as a pretreatment for convective air-drying evaluating the benefits of the pretreatment to the drying process and to the nutritional quality of the dried product. Mangoes were subjected to pulses of UV light. The pretreated samples were further dried in a convective ovendrier until $90 \%$ of the initial water content has been removed. Drying kinetics, water apparent diffusivity, vitamin $B$, vitamin $C$ content and total carotenoids content were analyzed. Pulsed UV light showed to be an interesting pretreatment for mangoes given the higher nutritional content of the dried product.
\end{abstract}

Keywords: mango; drying; ultraviolet; vitamins; kinetics. 


\section{Introduction}

New food product development should focus on retaining as much of the naturally occurring vitamin content as possible, on increasing the availability of vitamins, and on reducing the appearance of undesirable breakdown products. Factors that play a role in the degradation of vitamins during food processing include temperature, air, light, moisture content, water activity, $\mathrm{pH}$ and enzymes related to the food spoilage [1]. Air-drying reduces the moisture content of the product decreasing the effect of one of the primary factors, the water activity, that determines the rate of vitamin deterioration by several biochemical reactions [1]. However, air-drying may increase vitamin loss by increasing the food exposition to high temperature for a long period of time.

Pulsed ultraviolet (UV) light processing of foods is a nonthermal technology used to enhance the quality and safety of foods. Its uses range from sanitization of foods (primary use) to extension of shelf life of fruits and vegetables, and enhancement of phytochemical content in fruits [2,3]. UV treatment is currently in use by the industry especially in sanitization of fruit and vegetables. Several studies reported that UV light also contributed towards the enhancement of the nutritional quality of food products, enhancing vitamin content, total carotenoids content, antioxidant capacity and other phytochemical properties [3-5].

Despite the importance of quality parameters in dried foods, most published studies are limited to determining the drying rate and some diffusional aspects and only few studies have focused on evaluating the changes on product quality after drying. In this work, pulsed UV light was applied as a pretreatment prior to air-drying for the drying of mangoes. The influence of the pretreatment and the drying process on vitamins B1, B3, B5, B6 and C, total carotenoids as well as its influence on the effective water diffusivity were evaluated.

\section{Materials and Methods}

\subsection{Preparation of samples}

Mangoes (Mangifera indica L. var. Tommy Athikins) were bought from the local market (Fortaleza, Brazil). Only fruits with same maturity stage were used. Cubic samples (side 10 $\mathrm{mm}$ ) were obtained using a household tool from the mango flesh. The moisture content was determined by oven drying at $110^{\circ} \mathrm{C}$ until constant weight (24h).

\subsection{Pulsed UV light pre-treatment}

The pre-treatment was carried out in a pulsed UV light equipment (SteriBeam model Xe Matric A, Germany) equipped with a xenon flash lamp (19 cm). The samples (12 g $\pm 1 \mathrm{~g})$ were placed inside the treatment chamber of the equipment $(20 \mathrm{~cm}$ wide $\times 14 \mathrm{~cm}$ deep $\times 12$ $\mathrm{cm}$ high) and subjected to UV light pulses. The samples were subjected to 10, 20, 30, 40 and 50 pulses of UV light. Each pulse corresponded to a fluence (energetic density) of 0.36 $\mathrm{J} / \mathrm{cm}^{2}$ and were delivered in $250 \mu \mathrm{s}$. All experiments were carried out in triplicate. 


\subsection{Air drying}

Drying kinetics were carried out in a conventional convective oven-drier. Air-drying experiments with mango samples were carried out at $0.5 \mathrm{~m} / \mathrm{s}$ (air velocity) and $60{ }^{\circ} \mathrm{C}$ (temperature). The drying experiments were conducted in triplicates and completed when the samples lost $90 \%$ of the initial weight. For each experiment, $25 \pm 1 \mathrm{~g}$ cubes of mangoes were distributed over a custom sample holder inside the drying chamber.

The air-drying kinetics of mangoes was modeled assuming diffusion-controlled mass transfer. Only the falling-rate period (diffusion-controlled mass transfer period) was considered because the constant-rate period (heat transfer-controlled mass transfer period) was not observed. The model considered the solution of Fick's second law for cubic shaped samples (Equation 1) [6].

$$
W(t)=W_{e q}+\left(W_{\text {crit }}-W_{e q}\right)\left[\sum_{n=0}^{\infty} \frac{8}{(2 n+1)^{2} \pi^{2}} \exp \left(-\frac{D(2 n+1)^{2} \pi^{2} t}{4 a^{2}}\right)\right]^{3}
$$

\subsection{Determination of vitamins}

To determine the vitamins of the B complex, $1 \mathrm{~g}$ of fruit was homogenized with $6 \mathrm{~mL}$ of distilled water for 2 min using a cell homogenizer (Ultraturrax IKA model T25). The vitamins were extracted by adding sulfuric acid $0.25 \mathrm{M}(1 \mathrm{~mL})$ to the sample, which was heated for $30 \mathrm{~min}$ in a water bath $\left(70^{\circ} \mathrm{C}\right)$. After cooling in an ice bath, the $\mathrm{pH}$ was adjusted to 4.5 using a $0.5 \mathrm{M}$ sodium hydroxide solution. The sample was centrifuged at $8400 \times \mathrm{g}$ for $10 \mathrm{~min}$. The supernatant was collected and analyzed spectrophotometrically at 215 (Vitamin B5), 254 (Vitamin B1), 265 (Vitamin B3) and 716 (Vitamin B6) nm using water as blank. All analyses were carried out in triplicate and results were expressed as the vitamin gain/loss using the fresh fruit as reference.

\subsection{Determination of total carotenoid content}

The carotenoids were extracted milling $1 \mathrm{~g}$ of mango sample with $6 \mathrm{~mL}$ of distilled water in a cell homogenizer (Ultraturrax IKA model T25). Hexane $(5 \mathrm{~mL})$ was added and the mixture was vigorously stirred in a vortex for $1 \mathrm{~min}$. The supernatant (hexane phase) containing the lipid fraction was collected and analyzed spectrophotometrically at $452 \mathrm{~nm}$ to determine the total carotenoids content, using hexane as blank. All analyses were carried out in triplicate.

\section{Results and Discussion}

\subsection{Pulsed UV light pretreatment}

Mangoes presented an initial moisture content of $84.2 \pm 0.4 \mathrm{~g}$ water/100 $\mathrm{g}$ fresh fruit (wet basis). The moisture content of the samples decreased during the UV light pretreatment, 
reducing the initial moisture content by 4.3 to $15.9 \%$. Figure 1 presents the water loss observed as a function of the energetic density that was applied.

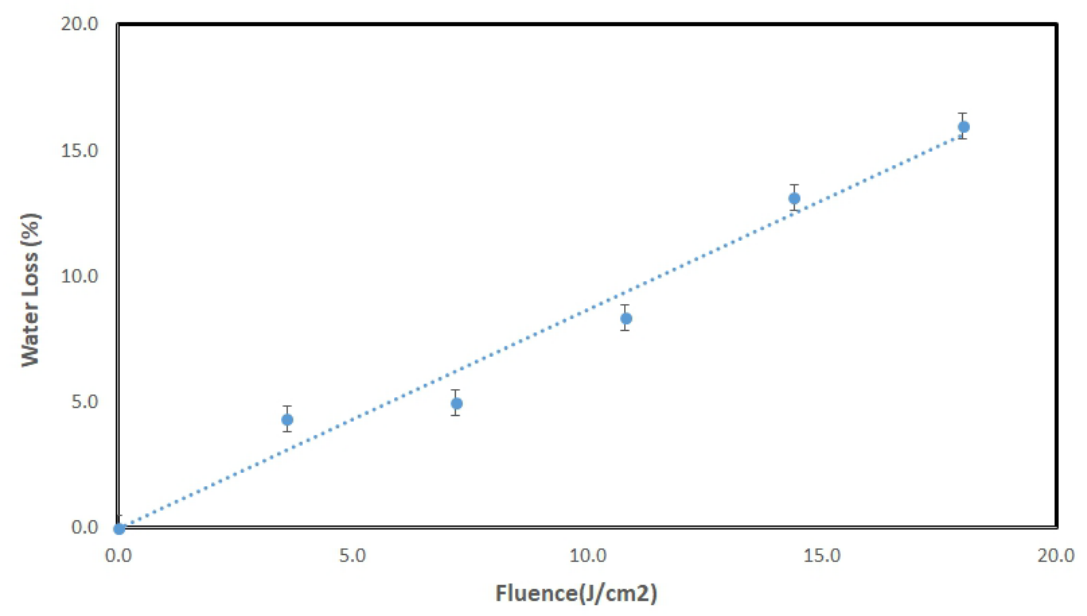

Fig. 1 Water loss during the pulsed UV light pretreatment as a function of the UV dosage applied to the samples.

Overall, pulsed UV light pretreatment contributed to an initial reduction of the moisture content of the samples.

\subsection{Air-drying experiments}

Figure 2 presents the experimental drying kinetics of mangoes obtained with and without application of pulsed UV light. Drying kinetics of mangoes presented the typical behavior observed for other fruits [7]. Only the falling rate period was observed during the drying process. Drying was carried out until 95\% of the initial moisture was removed, corresponding to a final moisture content of $0.08 \pm 0.01 \mathrm{~g}$ water/g dry matter.

The water apparent diffusivities in mangoes were calculated using Equation 1. The results are presented in Table 1. The diffusion model used in this work was adequate for describing the drying kinetics of mangoes cubes under the different experimental conditions, presenting $\mathrm{R}^{2}$ values between 0.986 to 0.995 .

Despite the initial reduction in moisture content attained during the pretreatment, the pulsed UV light pretreatment did not reduce the air-drying time. In fact, the samples took between 2 and 16\% more time to dry than the untreated sample. It must be stated that the required air-drying time to reduce $80 \%$ of the initial moisture content was statistically similar among the sample not subject to pretreatment and the samples pretreated with a total energetic density above $10.8 \mathrm{~J} / \mathrm{cm}^{2}$. 


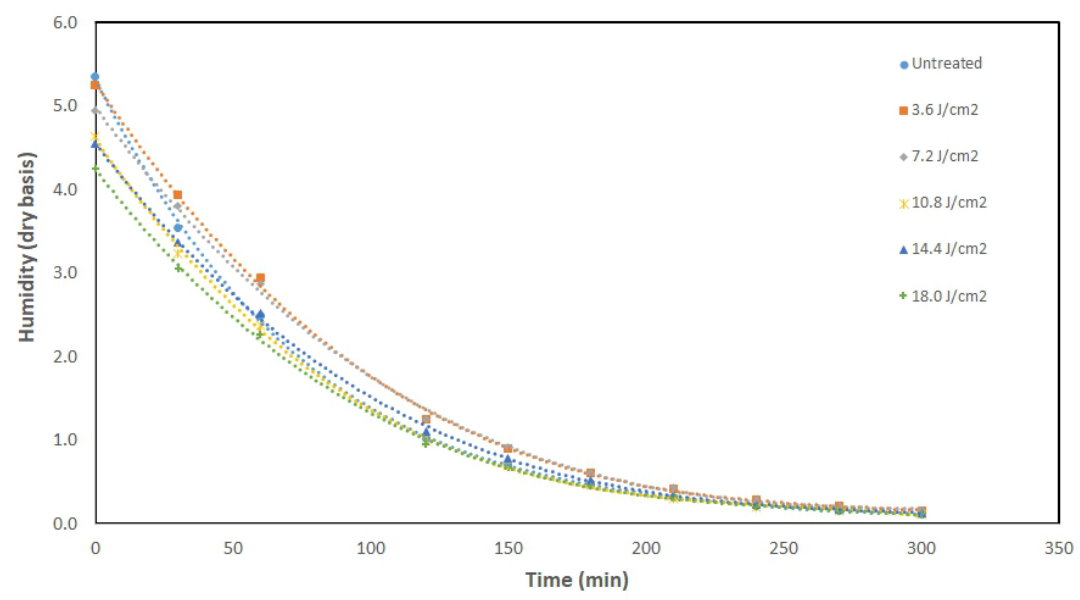

Figure 2. Moisture content (in dry basis) as a function of drying time for the samples with and without pulsed UV light pretreatment.

Table 1. Water apparent diffusivity of mangoes subjected to pulsed UV light pretreatment.

\begin{tabular}{llll}
\hline $\begin{array}{l}\text { Total energetic } \\
\text { density applied } \\
\left(\mathrm{J} / \mathrm{cm}^{2}\right)\end{array}$ & $\begin{array}{l}\text { Number of UV light } \\
\text { pulses } \\
(\#)\end{array}$ & $\begin{array}{l}\text { Apparent diffusivity } \\
\left(10^{7} \mathrm{~m}^{2} / \mathrm{min}\right)\end{array}$ & $\mathrm{R}^{2}$ \\
\hline 0.0 & 0 & $4.37 \pm 0.38^{\mathrm{a}}$ & 0.995 \\
3.6 & 10 & $3.90 \pm 0.03^{\mathrm{b}}$ & 0.986 \\
7.2 & 20 & $3.76 \pm 0.09^{\mathrm{b}}$ & 0.986 \\
10.8 & 30 & $4.26 \pm 0.12^{\mathrm{a}}$ & 0.987 \\
14.4 & 40 & $3.92 \pm 0.27^{\mathrm{a}}$ & 0.988 \\
18.0 & 50 & $4.10 \pm 0.01^{\mathrm{a}}$ & 0.991 \\
\hline
\end{tabular}

As such, if the main objective of the pretreatment is to reduce the air-drying time, the pulsed UV light pretreatment would not be a proper technology to achieve this goal.

\subsection{Vitamins and carotenoids content}

The drying process decreased the content of vitamins and total carotenoids in mangoes. The retention of vitamins and total carotenoids depended on the vitamin, the use or not of pulsed UV light pretreatment and the intensity of the pretreatment.

Figure 3 presents the retention of vitamins and total carotenoids for non-pretreated mangoes subjected to air-drying. Total carotenoids content presented the lowest retention ratio (24.6 
\%), while vitamin B6 presented the highest retention ratio (97.7 \%). The changes observed in Figure 3 represents the effect of heating in the vitamins and total carotenoid content.

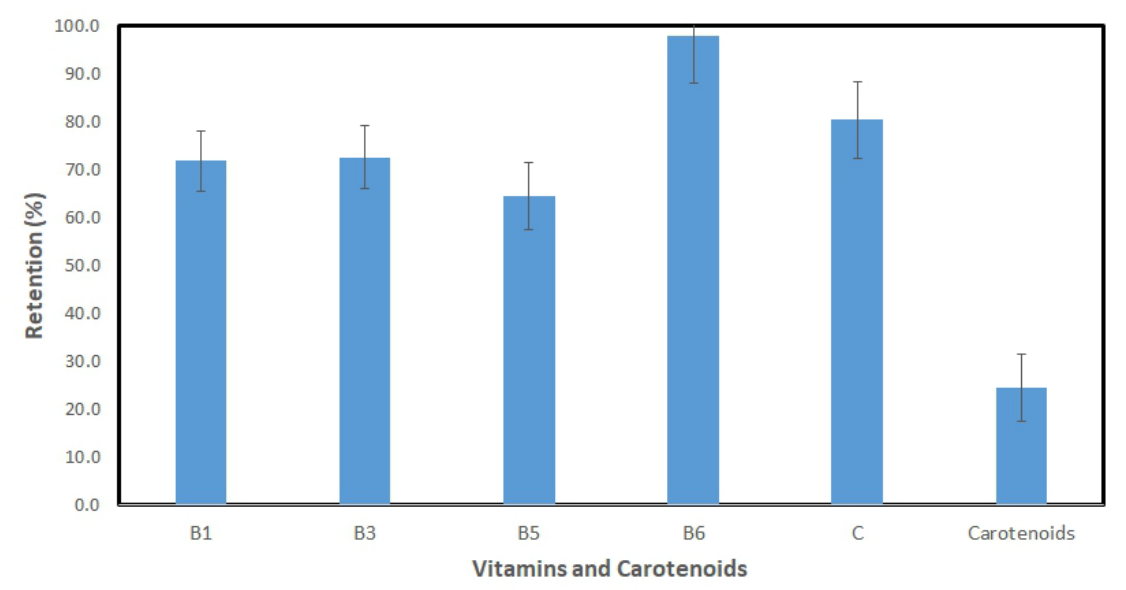

Figure 3. Retention of vitamins and carotenoids in mangoes after air-drying without $U V$ pretreatment.

The pulsed UV light pretreatment showed a positive effect on vitamins B1, B3 and B5 when up to $7.2 \mathrm{~J} / \mathrm{cm} 2$ where applied to the samples. Under such conditions, the dried product would present between 10.0 to $28.8 \%$ more vitamins B1, B3 and B5 than the untreated dried product. Above the $7.2 \mathrm{~J} / \mathrm{cm} 2$ level, the effect of the pretreatment is statistical non-significant (at a 95\% confidence level), due to a probable degradation of these vitamins by UV light.

Vitamin B1 (thiamin) is the most thermolabile among the B vitamins, so degradation due to thermal effect was expected [8] and was confirmed by the experimental data. The effect of pulsed UV light seemed to be mainly positive and a trend of higher retention of vitamin was observed. The high energy of the pulsed light treatment may have broken the bound of the phosphorylated vitamin, changing it to its free and bioavailable form, thus resulting in a slightly higher content of this vitamin in the treated dried product.

The retention of vitamins B3 and B5 followed a similar trend. As with vitamin B1, the energy delivered by pulsed UV light might have broken the bound between the vitamin and its bounds, releasing an amount of vitamin that otherwise would be unavailable. This is plausible because vitamins, nucleotides and coenzymes absorbs UV energy and could use this energy to break the chemical bond between vitamins and nucleotides, and vitamins and coenzymes. The energy absorbed from pulsed UV light causes physical damages to membranes and other structures due to the release of chemical and physical bonds in these structures [9]. Thus, the same energy that is responsible for bond breaking damage in microorganisms can be responsible for the bond breaking reactions that release the bonded vitamins and transform it in free and bioavailable vitamins. 
The pulsed UV light pretreatment had a significant negative effect on vitamin B6. The retention of vitamin B6 that was at $97.7 \%$ for the dried untreated mango, dropped to retentions levels between 48 to $61 \%$. This vitamin is considered light-sensitive. As such, both UV light and visible light released by the pulses have degraded this vitamin resulting in a significant reduction in its content when the UV pretreatment was applied.

The UV pretreatment did not change significantly the amount of vitamin $C$ in the dried product. The pretreatment was only statistically different (at a 95\% level of confidence) when a fluence of $12 \mathrm{~J} / \mathrm{cm}^{2}$ was applied. Under this condition, the amount of vitamin $\mathrm{C}$ was $28.6 \%$ higher than the untreated sample. Vitamin C is considered a light sensitive vitamin that present high UV light absorbance in the germicidal range (215 to $260 \mathrm{~nm}$ ) but does not absorb significant light above $300 \mathrm{~nm}$. The equipment used in our experiments releases light in a spectrum between 310 and $400 \mathrm{~nm}$, thus it has not affected the vitamin $\mathrm{C}$ content of the mangoes samples.

The UV pretreatment increased the total carotenoids when a fluence up to $12 \mathrm{~J} / \mathrm{cm}^{2}$ was applied. Overexposure to high intensity UV light tended to degrade the carotenoids. The increase in the total carotenoid content can be attributed to an alteration of the carotenoidbinding protein with a consequent increase in availability of free carotenoids, which has been reported previously for mango juice subjected to UV light treatment [10].

\section{Conclusions}

Pulsed UV light decreased the initial moisture content of the mangoes samples but did not increase the effective water diffusion of mangoes nor reduced its drying time. The application of pulsed UV light increased the availability of vitamins B1, B3, and B5, vitamin $C$ and carotenoids in the dried product. The light sensible vitamin B6 degraded significantly when compared to the untreated dried mango samples. Overexposure to pulsed UV light (dosages greater than $15 \mathrm{~J} / \mathrm{cm}^{2}$ ) degraded all vitamins and carotenoids, thus the UV dosage must be carefully optimized. Pulsed UV light can be a potential pretreatment for drying fruits given its simplicity, rapid application and because it confers better nutritional quality to dried mangoes.

\section{Acknowledgements}

The authors thank the financial support of the Brazilian funding agencies CAPES and CNPq.

\section{References}

[1] G.F.M. Ball, Vitamins in foods: analysis, bioavailability, and stability, CRC Press, Boca Raton, USA, 2006.

[2] T. Koutchma, I. Forney, C. Moraru, Ultraviolet light in food technology: principles and applications, CRS Press, Boca Raton, USA, 2009. 
[3] R. V. Tikekar, R.C. Anantheswaran, L.F. LaBorde, Ascorbic acid degradation in a model apple juice system and in apple juice during ultraviolet processing and storage, J. Food Sci. 76 (2011) 62-71.

[4] T. Koutchma, UV light for processing foods, Ozone-Science Engeneering. 30 (2008) 93-98.

[5] E. Cantos, C. Garcia-Viguera, S. Pascual-Teresa, F.A. Tomas-Barberan, Effect of postharvest ultraviolet radiation on resveratrol and other phenolics of cV. Napoleon table grapes, J. Agric. Food Chem. 48 (2000) 4606-4612.

[6] J. Crank, The mathematics of diffusion, 2nd ed., Oxfort University Press, Glasgow, 1975.

[7] F.A.N. Fernandes, S. Rodrigues, C.L. Law, A.S. Mujumdar, Drying of Exotic Tropical Fruits: A Comprehensive Review, Food Bioprocess Technol. 4 (2010) 163185. doi:10.1007/s11947-010-0323-7.

[8] K.T.H. Farrer, The thermal destruction of vitamin B1 in foods, Adv. Food Res. 6 (1955) 257-311.

[9] K. Krishnamurthy, J.C. Tewari, J. Irudayaraj, A. Demirci, Microscopic and spectroscopic evaluation of inactivation of Staphylococcus aureus by pulsed UV light and infrared heating, Food Bioprocess Technol. 3 (2010) 93-104.

[10] V. Santhirasegaram, Z. Razali, D.S. George, C. Somasundram, Comparison of UV-C treatment and thermal pasteurization on quality of chokanan mango (Mangifera indica L.) juice, Food Bioprocess Technol. 94 (2015) 313-321. 